\title{
Investigation of ultrafast dynamics of CdTe quantum dots by femtosecond fluorescence up-conversion spectroscopy*
}

\author{
Yao Guan-Xin(姚关心) $\left.{ }^{\mathrm{a}) \mathrm{b}}\right)$ ， Lü Liang-Hong(吕良宏 $)^{\mathrm{b})}$ ， Gui Mei-Fang(桂美芳) ${ }^{\mathrm{c}}$ ， \\ Zhang Xian-Yi(张先炏炎 $)^{\mathrm{b})}$, Zheng Xian-Feng(郑贤锋 $)^{\mathrm{b})}$, Ji Xue-Han(季学韩 $)^{\mathrm{b})}$, \\ Zhang Hong(张 宏 $)^{\mathrm{d}) \dagger}$, and Cui Zhi-Feng(崔执风) ${ }^{\mathrm{a}) \mathrm{b}) \ddagger}$ \\ a) Anhui Institute of Optics and Fine Mechanics, Chinese Academy of Sciences, Hefei 230031, China \\ b) Institute of Atomic and Molecular Physics, Anhui Normal University, Wuhu 241000, China \\ c) College of Chemistry and Materials Science, Anhui Normal University, Wuhu 241000, China \\ d) Van't Hoff Institute for Molecular Sciences, University of Amsterdam, Science Park 904, 1090 GD Amsterdam, The Netherlands
}

(Received 11 February 2012; revised manuscript received 7 April 2012)

\begin{abstract}
The ultrafast carrier relaxation processes in CdTe quantum dots are investigated by femtosecond fluorescence upconversion spectroscopy. Photo-excited hole relaxing to the edge of the forbidden gap takes a maximal time of $\sim 1.6 \mathrm{ps}$ with exciting at $400 \mathrm{~nm}$, depending on the state of the photo-excited hole. The shallow trapped states and deep trap states in the forbidden gap are confirmed for CdTe quantum dots. In addition, Auger relaxation of trapped carriers is observed to occur with a time constant of $\sim 5 \mathrm{ps}$. A schematic model of photodynamics is established based on the results of the spectroscopy studies. Our work demonstrates that femtosecond fluorescence up-conversion spectroscopy is a suitable and effective tool in studying the transportation and conversion dynamics of photon energy in a nanosystem.
\end{abstract}

Keywords: CdTe quantum dots, femtosecond fluorescence up-conversion spectroscopy, ultrafast dynamics, nanomaterial

PACS: $78.47 . \mathrm{N}-$, 78.67.Hc

DOI: $10.1088 / 1674-1056 / 21 / 10 / 107801$

\section{Introduction}

The quantum dots (QDs) are quasi zerodimensional nanomaterials which have aroused a lot of research interest in the last decade. Through size controlling, the band-gap energy of QD can be tuned in a broad spectral range and carrier relaxation paths can be manipulated, such as, trapping, radiative decay, and nonradiative decay. ${ }^{[1-5]}$ Compared with other semiconductor materials such as CdSe and CdS, CdTe has a great Bohr radius $(7.5 \mathrm{~nm})$ and a small band gap $(1.5 \mathrm{eV})$ and thereby exhibits strong quantum confinement effects. ${ }^{[6]}$ Due to the strong confinement effects of the carriers photo-generated in semiconductor nanoparticles, CdTe has strong nonlinear optical characteristics, which is an advantage in a variety of applications, such as optoelectronics, biophysics, highly efficient light-emitting nanocrystalline devices, infrared detectors, electronic communications, and solar cells. ${ }^{[7-15]}$

As far as ultrafast spectroscopy is concerned, recent studies of CdTe have focused on the complex behaviour of carrier relaxation..$^{[9,16-19]}$ Dimitrov et al. ${ }^{[16]}$ observed quantum beats in the pump-probe spectra of aqueous CdTe QDs after the resonant excitation of phonon wave packets with femtosecond pulses, and their results revealed that neither the exciton-phonon coupling strength nor the optical phonon frequencies is a function of the size of the dots. Kaniyankandy et al. ${ }^{[9]}$ reported on the ultrafast charge carrier relaxation dynamics of mercaptopropionic acid capped CdTe quantum QDs using femtosecond transient absorption spectroscopy, where the particles were excited at $400 \mathrm{~nm}$ and the transients were monitored in a range from the visible to near IR.

*Project supported by the National Natural Science Foundation of China (Grant Nos. 11074003 and 20973001) and the Key Program of Educational Commission of Anhui Province of China (Grant No. KJ2010A132).

${ }^{\dagger}$ Corresponding author. E-mail: h.zhang@uva.nl

$\ddagger$ Corresponding author. E-mail: zfcui@mail.ahnu.edu.cn

(C) 2012 Chinese Physical Society and IOP Publishing Ltd

http://iopscience.iop.org/cpb http://cpb.iphy.ac.cn 
They found that the cooling times of the second and first excitonic states are $150 \mathrm{fs}$ and $500 \mathrm{fs}$, respectively, dependent nonlinearly on size and that electron and hole trapping times were about 700 fs and 1 ps, respectively, in CdTe QD. Jin et al. ${ }^{[17]}$ synthesized the aqueous CdTe QDs by the electrostatic reaction method, and investigated the optical properties of CdTe QDs by the femtosecond time-resolved luminescence technique in non-resonant spectral region. They reported that the short-lived band-edge excitonic emission is independent of the detection wavelength, and long-lived species become even longer with the increase of detection wavelength, which indicates the size dependence of surface excitonic emission. In addition, ultrafast carrier relaxation dynamics have been investigated on the $\mathrm{CdTe}$ for core/shell structures in the fs-regime, such as CdSe/CdTe tetra-pod hetero-nanostructures and CdSe/CdTe donor-acceptor. ${ }^{[18,19]}$ Despite these efforts, the charge carrier dynamics of CdTe are not completely clear yet.

In this paper, we report on the electron and hole relaxation dynamics of $\mathrm{CdTe}$ QDs in water, which are investigated using femtosecond fluorescence upconversion spectroscopy. The time-resolved emission spectrum is more specific than its absorption counterpart in the sense that the former involves primarily the emission-related states while the latter contains all the states in resonance with the probing frequency. Our goal is to acquire a complete picture of the relaxation dynamics of photoexcited carriers, which is not only important from a fundamental research point of view, but also essential for the application of II-VI semiconductor nanomaterials. Based on the results, several ultrafast processes are distinguished.

\section{Experiments}

\subsection{Preparation and purification of TGA-coated CdTe QDs}

The CdTe QDs were prepared following the method described elsewhere. ${ }^{[20-22]} 0.2864 \mathrm{~g}$ (1.25 mmol) $\mathrm{CdCl}_{2} \cdot 2.5 \mathrm{H}_{2} \mathrm{O}$ was dissolved in $100 \mathrm{~mL}$ of double-distilled water, and 3.0-mmol thioglycolic acidstabilized (TGA) was added under stirring, followed by $\mathrm{pH}$ adjustment to 11.2 by a dropwise addition of 1 $\mathrm{M} \mathrm{NaOH}$ solution. The solution was deoxygenated by bubbling $\mathrm{N}_{2}$ through the solution for $40 \mathrm{~min}$. Freshly prepared, oxygen-free NaHTe was then injected into the above solution with stirring. The molar ratio of $\mathrm{Cd}^{2+} /$ TGA/HTe- was 1:2.4:0.5. The resulting mixture was heated at $100{ }^{\circ} \mathrm{C}-120{ }^{\circ} \mathrm{C}$ and refluxed for different amounts of time to grow the particles to the desired size.

\subsection{Femtosecond fluorescence up- conversion setup}

The schematic representation of the home-made femtosecond fluorescence up-conversion setup is shown in Fig. 1. Specifically, a mode-locked Ti:sapphire laser (Micra-5, Coherent, USA) was optically pumped by a frequency doubled Nd: $\mathrm{YVO}_{4}$ laser (Verdi, Coherent, USA), to produce pulses, at a wavelength of $800 \mathrm{~nm}$ with a repetition rate of $82 \mathrm{MHz}$. Amplification was realized by means of a Ti:sapphire regenerative amplifier system (Legend Elite-USP-1K-HE, Coherent, USA) pumped by an Nd:YLF laser (Evolution-30, Coherent, USA), which generated a pulse train of approximately $3.0 \mathrm{~mJ} /$ pulse under a $1-\mathrm{kHz}$ repetition. The amplified pulses were split into two beams with one serving as pumping an optical parametric amplifier system (OPAS). The wavelength-tunable laser pulses from the OPAS were used to optically excite the sample. In the up-conversion experiment, the excitation wavelength was selected to be $400 \mathrm{~nm}$ with energy of about $8 \mu \mathrm{J}$ pulse $^{-1}$, and the duration of the excitation pulse was about 100 fs. The sample was always kept flowing to prevent the sample from being overheated. A 450-nm cut-off filter set behind the sample prevented the scattered laser and/or Raman light in the detection from interfering with each other. The second laser beam, the gating beam, first passed through an optical delay line. The up-conversion signal was generated by wave mixing of the gating beam with the laser-induced fluorescence in a 1-mm BBO crystal (type I phase matching conditions). The upconverted fluorescence light passed through a UG11 band-pass filter and focused onto the entrance slit of a monochromator outfitted with a photomultiplier. Detection of the output signal from the photomultiplier was realized by means of lock-in amplification. The system response time was determined from the crosscorrelation signal of the gate- and excitation-beams 
to be about 160 fs (FWHM). All the measurements were performed at room temperature in a CdTe QDs aqueous solution.

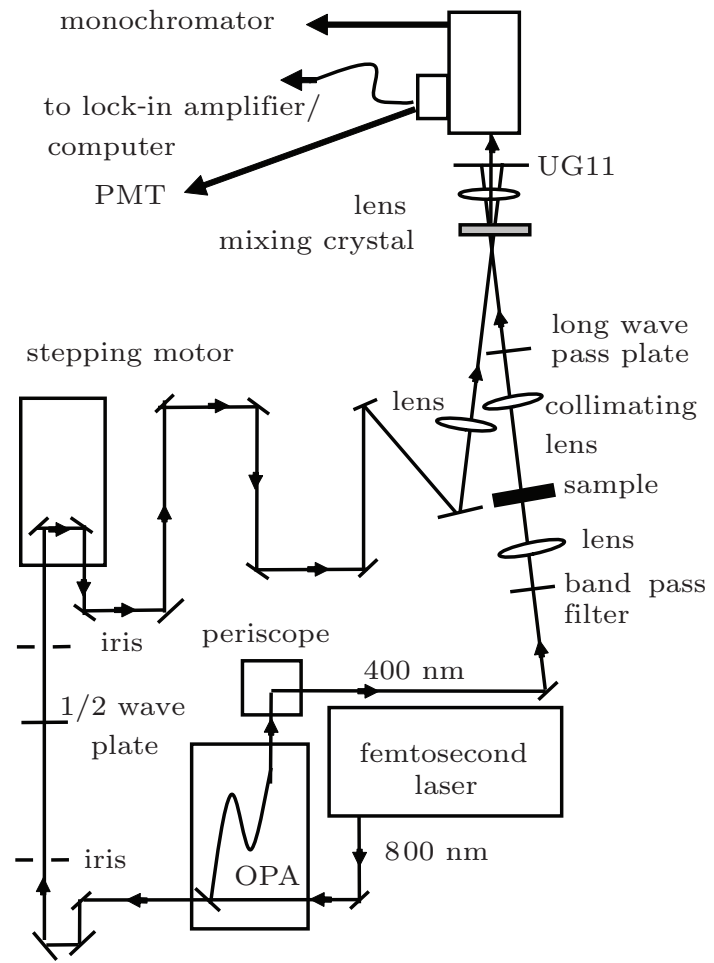

Fig. 1. Schematic representation of femtosecond fluorescence up-conversion setup.

\section{Results and discussion}

\subsection{Steady-state absorption- and PL spectra}

Figure 2 shows the steady-state absorption and photoluminescence (PL) spectra of CdTe QDs. A broad absorption band appears in the ultraviolet and visible region where the excitonic transition between the lowest electron state $\left(1 \mathrm{~S}_{1 / 2}\right)$ and hole state $\left(1 \mathrm{~S}_{3 / 2}\right)$ is determined to be $2.28 \mathrm{eV}(545 \mathrm{~nm})$. The average diameter of the CdTe QDs is thus deduced to be about $3.2 \mathrm{~nm}$ from the position of the first excitonic absorption peak. ${ }^{[23]}$ The PL spectrum peak is at $2.17 \mathrm{eV}(573 \mathrm{~nm})$ and the full width at half maximum (FWHM) is about $185 \mathrm{meV}$. The broadening of optical transition shown in the PL spectrum is attributed primarily to the inequality of the sample size. ${ }^{[24]}$ The emission peak is shifted from the first excitonic absorption peak by $110 \mathrm{meV}$. In addition, it is observed that the PL spectrum is asymmetric, the region of high energy has streaking conditions.

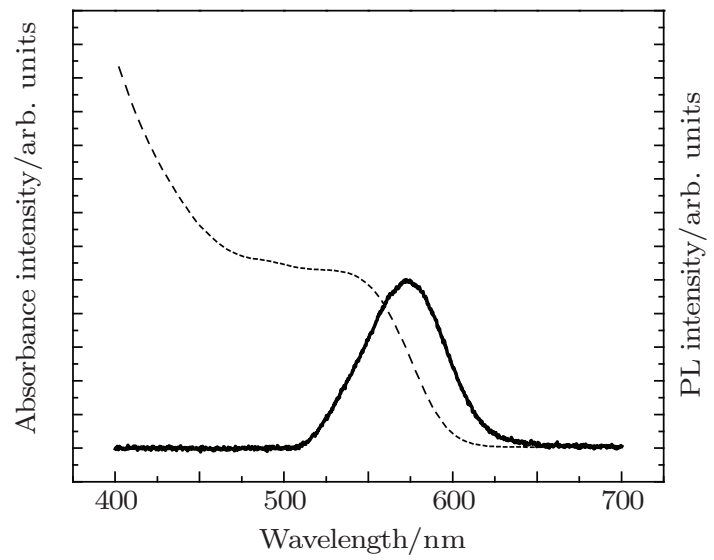

Fig. 2. The steady-state absorption spectrum (dashed line) and PL spectrum (solid line) with exciting at $400 \mathrm{~nm}$.

\subsection{Ultrafast up-conversion fluores- cence behaviour}

In order to study the inter-band and intra-band relaxation dynamics in CdTe QDs, time behaviours of the PL spectrum shown in Figs. 3(a)-3(d) are recorded at various wavelengths for a 10 -ps window. For comparison these decay curves are also normalized to the maximum value (Fig. 3(d)). It is observed in Fig. 3(d) that the rising and decaying part of the PL curve are gradually prolonged with the increase of the probing wavelength. In general, all the kinetic curves of PL shown in Fig. 3 can be reasonably well fitted to a multi-exponential function $I(t)=\sum A_{i} \exp \left(-t / \tau_{i}\right)$, with one exponential representing the rise component and the other two denoting the decay. The even longer components ( $\sim$ ns or longer $)$ are in this window fairly treated as being time-independent and represented by a fixed time constant in the order of ns. Results of fitting parameters are summarized in Table 1, where the rise time $\left(\tau_{\text {rise }}\right)$ demonstrates an increase from $0.37 \mathrm{ps}$ to $1.57 \mathrm{ps}$ as the probing wavelength increases from $550 \mathrm{~nm}$ to $590 \mathrm{~nm}$. As far as the decay is concerned, although the time constants, i.e., about 100 fs and several ps, vary little with probing wavelength, the corresponding amplitudes change significantly. More specifically, the 100-fs component drops down whereas the 550-nm component becomes more pronounced when detecting at a longer wavelength. It is worth noting that the $\tau_{1}$ component completely disappears at $590 \mathrm{~nm}$. 

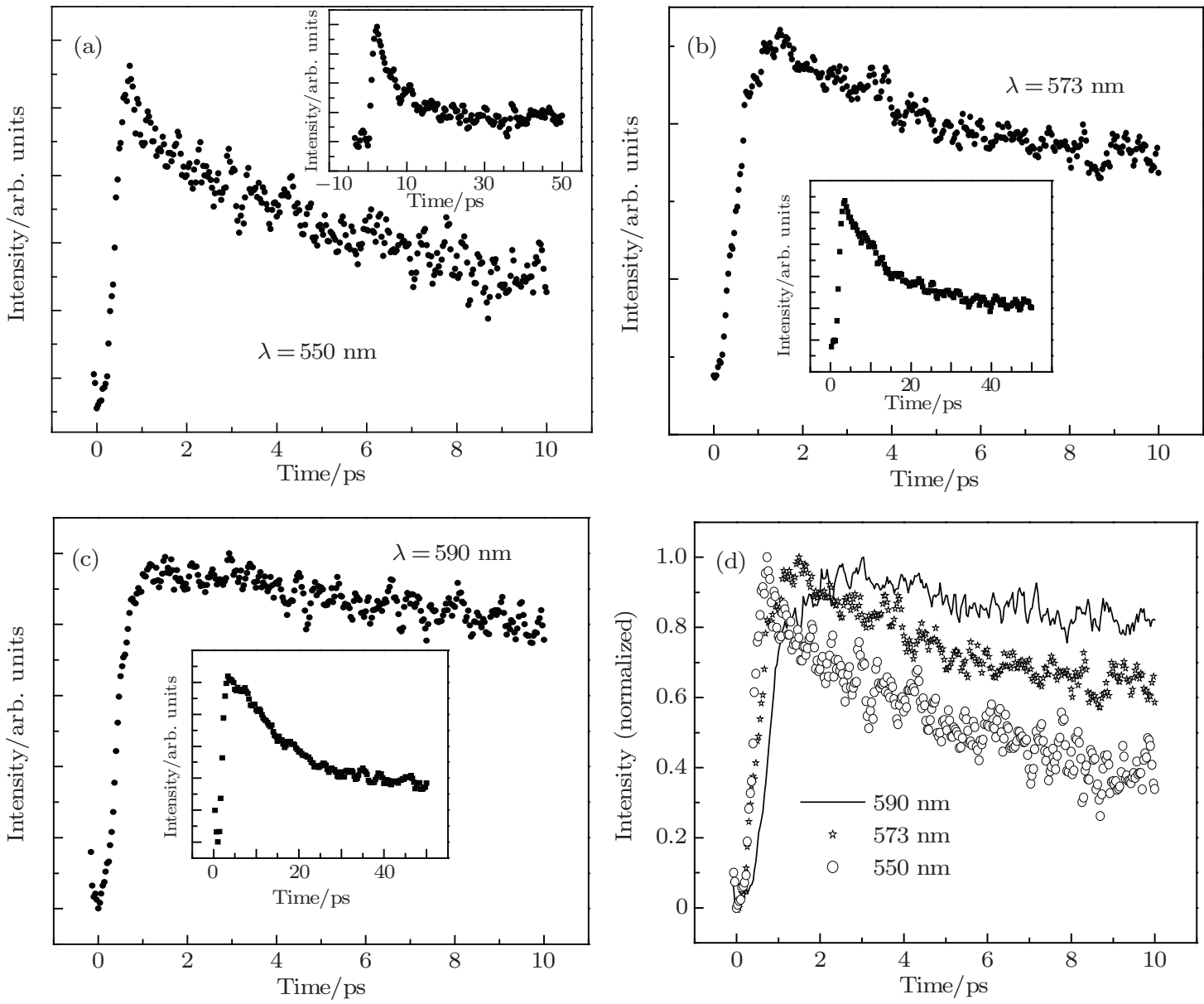

Fig. 3. Femtosecond fluorescence up-conversion transients of CdTe QDs at various wavelengths for a 10-ps window, with excitation at $400 \mathrm{~nm}$ and the inset showing the corresponding traces in a large window.

Table 1. Time constants of fluorescence up-conversion transient curves fitted with a multi-exponential decay described in the text under a 400-nm excitation.

\begin{tabular}{cccccccc}
\hline$\lambda / \mathrm{nm}$ & $\tau_{\text {rise }} / \mathrm{ps}$ & $\tau_{1} / \mathrm{ps}$ & $\tau_{2} / \mathrm{ps}$ & $\tau_{3}$ & $A_{1}$ & $A_{2}$ & $A_{3}$ \\
\hline 550 & 0.37 & 0.13 & 6.87 & $\sim \mathrm{ns}$ & 0.18 & 0.61 & 0.21 \\
573 & 0.88 & 0.11 & 4.43 & $\sim \mathrm{ns}$ & 0.05 & 0.38 & 0.57 \\
590 & 1.57 & & 4.53 & $\sim \mathrm{ns}$ & & 0.30 & 0.70 \\
\hline
\end{tabular}

\subsection{Discussion}

\subsubsection{Origins of red shift and asymmetry of the PL spectrum}

The relatively large Stokes shift of the PL peak may be related to several plausible reasons. ${ }^{[25-27]}$ The first one is the excitonic fine structure and strong carrier-phonon interaction, the second one is the presence of defect states (localized states) which are mixed with the band-edge emission in the spectrum, and the last one may be the effect of the electric field of many electron-hole pairs formed in each particle under a high pump intensity.
In our experiment, the average number of electron-hole pairs per CdTe particle $\langle n\rangle$ can be calculated by the following formulas: ${ }^{[1,23]}$

$$
\begin{aligned}
& \langle n\rangle=j_{p} \sigma_{0}, \\
& \sigma_{0}=A /\left(n_{q}^{*} L\right), \\
& A=C^{*} C L, \\
& C^{*}=10043(D)^{2.12},
\end{aligned}
$$

where $j_{p}$ is the excitation fluence, $\sigma_{0}$ is the $\mathrm{NC}$ absorption cross section at the pump wavelength, $A$ is the absorbance at the peak position of the first exciton absorption of CdTe, $n_{q}$ is the number density of nanocrystals, $L$ is the path length of the radiation beam used for recording the absorption spectrum (fixed at $1 \mathrm{~cm}$ ), $C^{*}$ is the extinction coefficient per mole of nanocrystals, $C$ is the molar concentration of $\mathrm{CdTe}$, and $D$ is the average diameter of $\mathrm{CdTe}$ $(3.2 \mathrm{~nm})$. In our measurement, the excitation energy density is adjusted to about $800 \mu \mathrm{J} / \mathrm{cm}^{2}$ at $400 \mathrm{~nm}$. 
From Eqs. (1)-(4), the $\langle n\rangle$ was obtained to be about 0.3. Therefore, the possibilities for multi-excitons and biexcitons to exist are very small, and the effect of the electric field of many electron-hole pairs in our experimental conditions can be excluded from the analysis of the large Stokes shift.

The fastest decay time constant $\tau_{1}$ is about $100 \mathrm{fs}$ and can be attributed to the trapping process of surface states, by viewing from the time order of magnitude. ${ }^{[28]}$ However, we observe this ultrafast decay component at all detection wavelengths except $590 \mathrm{~nm}$. Therefore, we tentatively believe that emission $<573 \mathrm{~nm}$ comes mainly from the luminescence of exciton state $\left(1 \mathrm{~S}_{3 / 2}(\mathrm{~h})-1 \mathrm{~S}(\mathrm{e})\right)$, and the contribution of the surface state turns non-negligible or even dominant when the probing wavelength is longer than $590 \mathrm{~nm}$.

These facts indicate that the Stokes shift should be ascribed to the presence of surface states which are mixed with the band-edge emission in spectrum, or the excitonic fine structure and strong carrier-phonon interaction (see Fig. 4), and the asymmetry of the PL spectra can be understood from the fact that the PL spectrum originates from different $\mathrm{e}-\mathrm{h}$ recombination mechanisms.

\subsubsection{Carrier relaxation dynamics in CdTe QD}

The ultrafast behaviour of the PL reflects the excited state dynamics. In the following we acquire, from the analysis of the femtosecond transients recorded by up-conversion technique, dynamic pictures of the interaction between carriers, between carriers and phonons, and between carriers and defects.

\subsubsection{Dynamic processes with the time constants of $\tau_{\text {rise }}$ and $\tau_{1}$}

The band gap of CdTe QD is about $2.28 \mathrm{eV}$ as obtained from Fig. 2. The excitation photon energy in our experiment is about $3.11 \mathrm{eV}$ which exceeds the band gap. As pointed out elsewhere, ${ }^{[29]}$ the electrons and the holes are mainly the injected $1 \mathrm{~S}(\mathrm{e})$ electron state and several hole states $\left(1 \mathrm{~S}_{3 / 2}(\mathrm{~h}), 2 \mathrm{~S}_{3 / 2}(\mathrm{~h})\right.$, and $\left.3 \mathrm{~S}_{3 / 2}(\mathrm{~h})\right)$ with the $400-\mathrm{nm}$ exciting pulse. It is claimed that the electrons of the excited state relax rapidly to the lowest electronic state $1 \mathrm{~S}(\mathrm{e})$ within 50 fs. ${ }^{[25]}$ This time is too short to be resolved in our experimental conditions, therefore the carrier relaxation processes we have observed should take place after the electron has relaxed to $1 \mathrm{~S}(\mathrm{e})$. The rise time that we obtain from the PL decay curves, can reveal the relaxation processes of the holes. As argued in Subsubsection 3.3.1, the values of the fastest decay time constant $\tau_{1}$ at $550 \mathrm{~nm}$ and $573 \mathrm{~nm}$ can be attributed to the trapping processes of surface states, and the PL at $590 \mathrm{~nm}$ is from the surface state. Here, the $\tau_{1}$ process can be thought as the rise process at $590 \mathrm{~nm}$, but the rise at $590 \mathrm{~nm}$ is about $1.57 \mathrm{ps}$, which is far larger than $\tau_{1}$. Thus the rise at $590 \mathrm{~nm}$ does not represent the surface trapping process. The result leads us to conclude that the rise detected at $590 \mathrm{~nm}$ originates from the hole relaxation dynamics since the PL depends not only on electrons, but also on holes. In general, the relaxation of holes takes much time compared with electrons due to the mass difference between the electron and hole. In order to illuminate intuitively the relaxation processes of holes, the scheme of electron and hole level energies in CdTe QD are drawn in Fig. 4, where the $1 \mathrm{~S}_{3 / 2}(\mathrm{~h})$ is split into several levels when the spin-orbit coupling is taken into account.

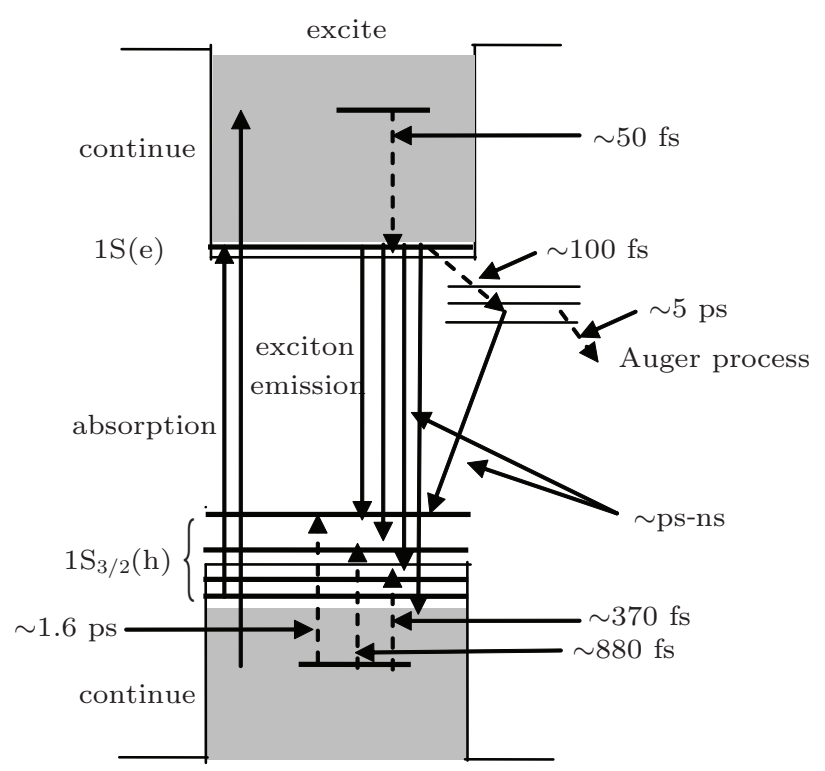

Fig. 4. Scheme of electron and hole level energies in CdTe.

When the sample is excited with 400-nm femtosecond pulses, the electrons are injected rapidly into the $1 \mathrm{~S}(\mathrm{e})$ in about $50 \mathrm{fs}$, and the holes are prepared in higher hole excited states. The rise time at $590 \mathrm{~nm}$ where the surface state emission is dominant makes it clear that the holes relax to the valence band edge from the initially populated hole states in about $1.6 \mathrm{ps}$. Compared with the rise time at $550 \mathrm{~nm}(0.37 \mathrm{ps})$, it 
is found that the hole relaxation rate is slow in this process. The reason is that the hole relaxation in the $1 \mathrm{~S}_{3 / 2}(\mathrm{~h})$ state needs phonons to assist energy transfer, and the multi-phonon relaxation process thus turns slower. This is in line with the report that the hole's final relaxation step in the CdSe $1 \mathrm{~S}_{3 / 2}(\mathrm{~h})$ state is relatively slow compared with the hole filling. ${ }^{[30]}$

\subsubsection{Dynamic process with time constants of $\tau_{2}$ and} $\tau_{3}$

From Table 1, it is noted that the electrons relaxing from $1 \mathrm{~S}(\mathrm{e})$ to the surface states have two components with time constants of $\tau_{2}$ and $\tau_{3}$, respectively. The existence of a ps component $\tau_{2}$ at $590 \mathrm{~nm}$, where surface states' emission is dominant, indicates that another energy loosing channel, other than the direct recombination of electrons with holes, must exist for the surface states. For this aspect, a deep trap state may be a good candidate. ${ }^{[31]}$ The surface state electrons relaxing to deep trap states could be realized with the aid of the Auger process (about $\sim \mathrm{ps}$ ).

The time constant $\tau_{3}$ represents all the recombinations slower than ns, including the intrinsic radiative recombination and the slow recombination of surface traps, as has been well studied. ${ }^{[32-34]}$

\section{Conclusions}

In this paper, the electron and hole relaxation dynamics in CdTe QDs are studied in an aqueous solution by femtosecond fluorescence up-conversion experiments. From the recorded PL kinetics, the time of the holes relaxing to the edge of CdTe valence band is determined to be about $1.6 \mathrm{ps}$. The shallow trap states and deep trap states are distinctly illustrated to exist. Auger relaxation of the trapped carrier is measured to occur in $\sim 5$ ps. These results can contribute to understanding the complicated photodynamics in quantum dot systems.

\section{References}

[1] Klimov V I 2000 J. Phys. Chem. B 1046112

[2] Efros Al L and Efros A L 1982 Sov. Phys. Sem. 16772

[3] Brus L E 1983 J. Chem. Phys. 795566

[4] Brus L E 1991 Appl. Phys. A 53465
[5] Alivisatos A P 1996 Science 271933

[6] Rajh T, Micic O I and Nozik A J 1993 J. Phys. Chem. 97 11999

[7] Wang Z, Li B, Zheng X, Xie J, Huang Z, Liu C, Feng L H and Zheng J G 2010 Chin. Phys. B 19027303

[8] Dzhafarov T D and Caliskan M 2007 J. Phys. D: Appl. Phys. 404003

[9] Kaniyankandy S, Rawalekar S and Ghosh H N 2010 Phys. Chem. Chem. Phys. 124210

[10] Deng Z T, Y Zhang, J C Yue and Q Wei 2007 J. Phys. Chem. B 11112024

[11] Li B, Feng L H and Zeng G G 2011 Chin. Phys. B 20 037103

[12] Medintz I L, Uyeda H T and Mattoussi H 2005 Nat. Mater. 4435

[13] Patrick T K, Svetlana S B and Janssen A J 2008 Nanotechnol. 19205602

[14] Hong W P and Park S H 2011 Chin. Phys. B 20098502

[15] Kumar S, Sharmaa S K and Husain M 2000 J. Phys. Chem. Solids 611809

[16] Dimitrov S D, Dooley C J and Fiebig T 2009 J. Phys. Chem. C 1134198

[17] Jin Q H, Wu W Z and Su W H 2009 J. Nanopart. Res. 11665

[18] Peng P, Milliron D J and Saykally R J 2005 Nano. Lett. 51809

[19] Dooley C J, Dimitrov S D and Fiebig T 2008 J. Phys. Chem. C 11212074

[20] Xia Y, Zhang T and Diao X C 2007 Chem. Lett. 36242

[21] Sagarzazu G, Kobayashi Y and Tamai N 2011 Phys. Chem. Chem. Phys. 133227

[22] Kuo Y C, Wang Q and Huang Q C 2008 J. Phys. Chem. C 1124818

[23] Yu W W, Qu L H and Guo W Z 2003 Chem. Mater. 15 2854

[24] Tang N Y, Ji Y L and Chen X S 2008 J. Funct. Mater. Dev. 14603

[25] Sanz M, Correa-Duarte M A and Douhal A 2008 J. Photochem. Photobiol. A 19651

[26] Burda C, Link S and El-Saye M A 1999 J. Phys. Chem. B 10310775

[27] Klimov V I, Haring P and Kurz H 1996 Phys. Rev. B 53 1463

[28] Padilha L A, Neves A A and Cruz C H 2004 Appl. Phys. Lett. 853256

[29] Wang H Y, Donegá C M and Glasbeek M 2006 J. Phys. Chem. B 110733

[30] Xu S, Mikhailovsky A A and Klimov V I 2002 Phys. Rev. B 65045319

[31] Liu B G, He C Y and Gao C X 2011 Phys. Status Solidi B 2481102

[32] Wang X Y, Yu W W and Xiao M 2003 Phys. Rev. B 68 125318

[33] Kapitonov A M, Stupak A P and Eychmu1ller A $1999 \mathrm{~J}$. Phys. Chem. B 10310109

[34] Mowbray D J and Skolnick M S 2005 J. Phys. D: Appl. Phys. 382059 